\title{
Corrections
}

\section{Correction: Pérez and Merchant, "The Synaptic Properties of Cells Define the Hallmarks of Interval Timing in a Recurrent Neural Network"}

In the article "The Synaptic Properties of Cells Define the Hallmarks of Interval Timing in a Recurrent Neural Network" by Oswaldo Pérez and Hugo Merchant, which appeared on pages 4186-4199 of the April 25, 2018 issue, an incorrect acknowledgment was listed. The authors apologize for the oversight. The corrected acknowledgment is as follows: "This work was supported by Consejo Nacional de Ciencia y Tecnología Grants 236836 and 196, and Programa de Apoyo a Proyectos de Investigación e Innovación Tecnológica Grant IN202317, Secretaria de Ciencia, Tecnología e Innovación to H.M. We thank Victor de Lafuente, Warren Meck, Fernando Peña, and Dobromir Dotov for their fruitful comments on earlier versions of the paper, Luis Prado and Raul Paulín for their technical assistance. Oswaldo Pérez is a doctoral student from Programa de Doctorado en Ciencias Biomédicas, Universidad Nacional Autónoma de México (UNAM) and received fellowship 204516 from CONACYT.” This correction does not affect the conclusions of the paper. The acknowledgments have been corrected on the online PDF version.

DOI:10.1523/JNEUROSCI.1689-18.2018

\section{Correction: Stalnaker et al., "Cholinergic Interneurons Use Orbitofrontal Input to Track Beliefs about Current State"}

In regards to the article, "Cholinergic Interneurons Use Orbitofrontal Input to Track Beliefs about Current State" by Thomas A. Stalnaker, Ben Berg, Navkiran Aujla, and Geoffrey Schoenbaum, which appeared on pages 6242-6257 of the June 8, 2016 issue, the authors discovered a bug in the MATLAB code that calculated the mean coefficient of variation (CV2) index for each neuron in the population. This index was used to identify putative cholinergic interneurons (CINs) as those with mean CV2 $<0.80$. The bug artificially lowered the mean CV2 by averaging a spurious -999 into the list of CV2 values for each pair of interspike intervals for each neuron. This only had a substantial effect on the small minority of neurons with a very low number of spikes (very low firing rates), which were therefore inaccurately identified as putative CINs. Upon finding this error, we recalculated the CV2 with corrected code and reanalyzed the resulting revised lists of CINs. The re-analyses showed qualitatively similar results to those reported in the original paper, including statistical significance.

Because the distribution of mean CV2 indices of putative medium spiny neurons (MSNs; defined by waveform and firing rate characteristics) were slightly shifted upward in the corrected dataset, we were also able to better distinguish putative CINs from putative MSNs. Therefore we identified a slightly higher CV2 criterion for putative identification of CINs $(<0.85)$, which still distinguished putative CINs from putative MSNs statistically and was still consistent with published data on confirmed CINs. When we reanalyzed the data using this revised criterion, we also found similar results to the original paper, again including statistical significance. The revised figures in this corrigendum are parallel to the figures in the original paper except that they use the corrected CV2 values and the revised putative CIN lists using the 0.85 criterion (Figs. 1-12). These revised lists excluded 30 of the 109 total CINs analyzed in the original paper (average baseline firing rate of these 30 neurons $=0.8$ spikes/s, average corrected CV2 $=1.08$ ). The original figure legends are also remade, with $n$ values and statistical values changed to reflect the re-analyses. The authors apologize for this error but reaffirm that the description and interpretation of the data reported in the article are not affected. 


\section{a Task Sequence}

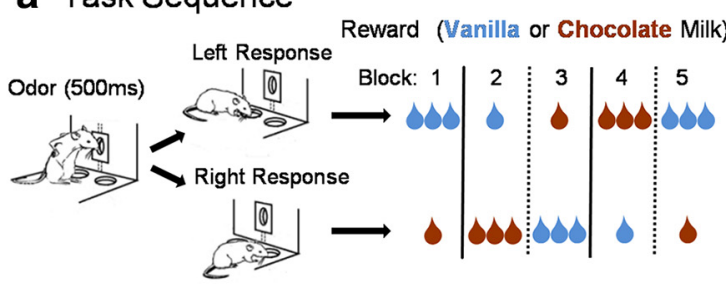

\section{b Unilateral OFC Lesions}

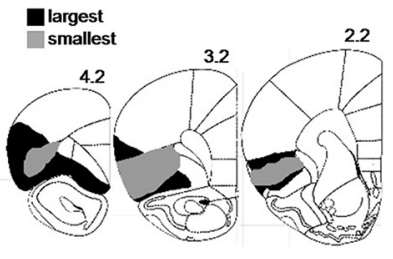

\section{Free-Choice, Number Block Switches}
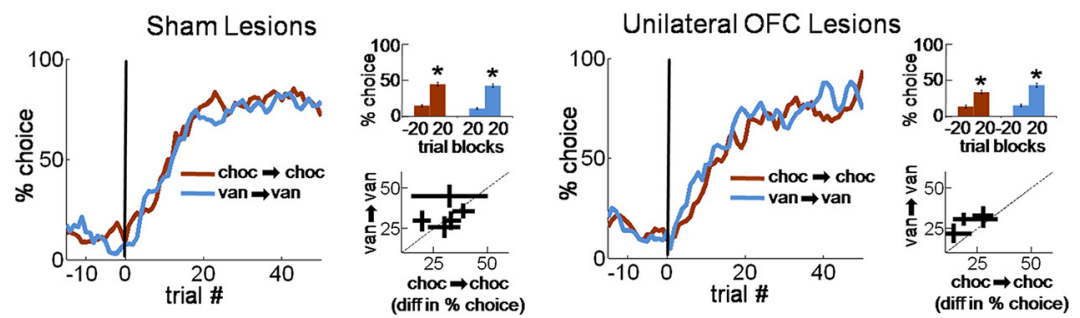

\section{d Free-Choice, Flavor Block Switches}
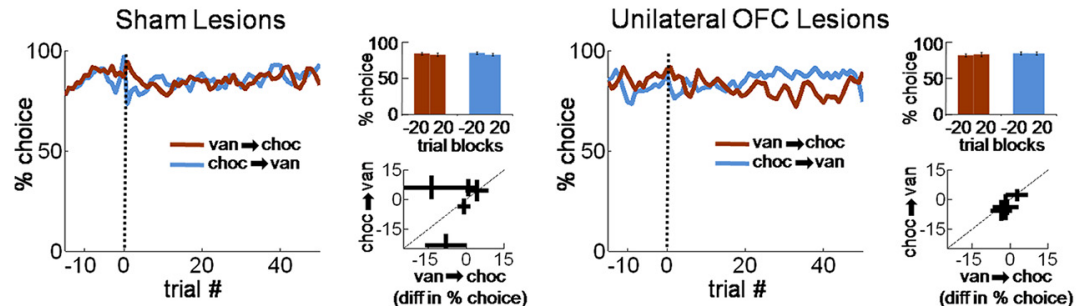

e Neural Dataset

Sham Group, DMS $(n=538)$

Lesion Group, DMS ( $n=793)$
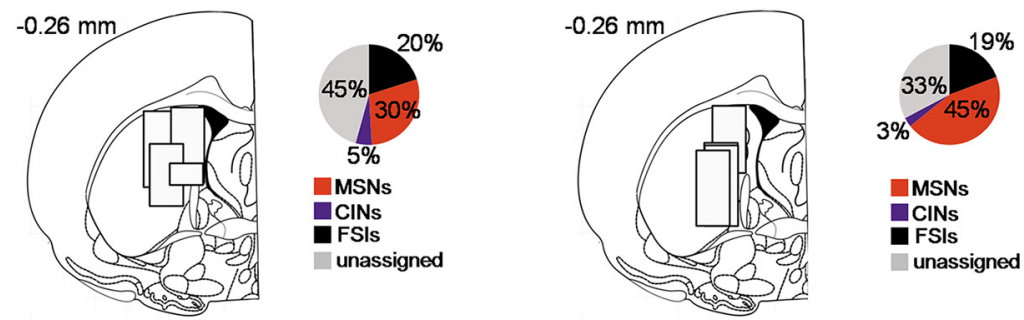

Sham Group, DLS $(n=624)$
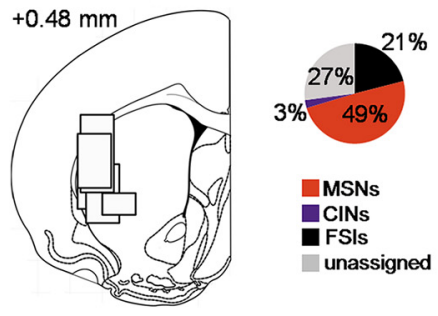

Lesion Group, DLS ( $n=759)$

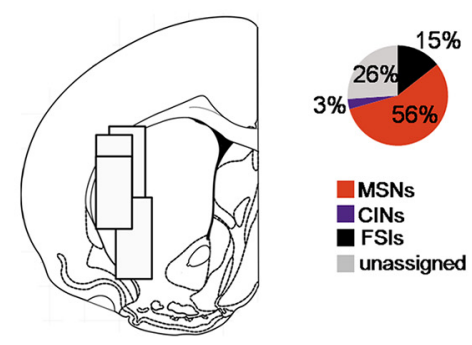

Figure 1. The task, lesions, behavior, and recording locations. The task ( $\boldsymbol{a}$ ) had four different blocks, or states, defined by the set of available response-reward contingencies. Trials began with an instructional odor, indicating a free-choice or forced-choice, after which rats responded at one of the two fluid wells for 1 or 3 drops of chocolate or vanilla milk. Reward contingencies were stable across blocks of $\sim 60$ trials, but at unsignaled transitions the number of drops or flavor changed on both sides (only 1 of the 4 possible block sequences is shown). Unilateral neurotoxic lesions of orbitofrontal cortex $(\mathrm{OFC})(\boldsymbol{b})$ were made in one group of rats (numbers are millimeters from bregma). Groups with sham lesions or unilateral lesions of OFC were similarly sensitive to changes in the number of drops $(\boldsymbol{c})$, and similarly insensitive to changes in the flavor $(\boldsymbol{d})$. Line figures show average trial-by-trial choice rates across transitions; bar graphs summarize these data by showing average choice rates in the last 20 trials of the previous block versus the first 20 of the new block; scatter plots show rat-by-rat difference scores (choice rate after minus choice rate before) with length of lines showing SEs. $\boldsymbol{e}$, The approximate locations of recordings and proportions of putative cell types in each of the four groups. The width of each box represents $1 \mathrm{~mm}$. FSI, Fast-spiking interneuron. ${ }^{*} p<0.001$. 


\section{a Cell Type Separation}
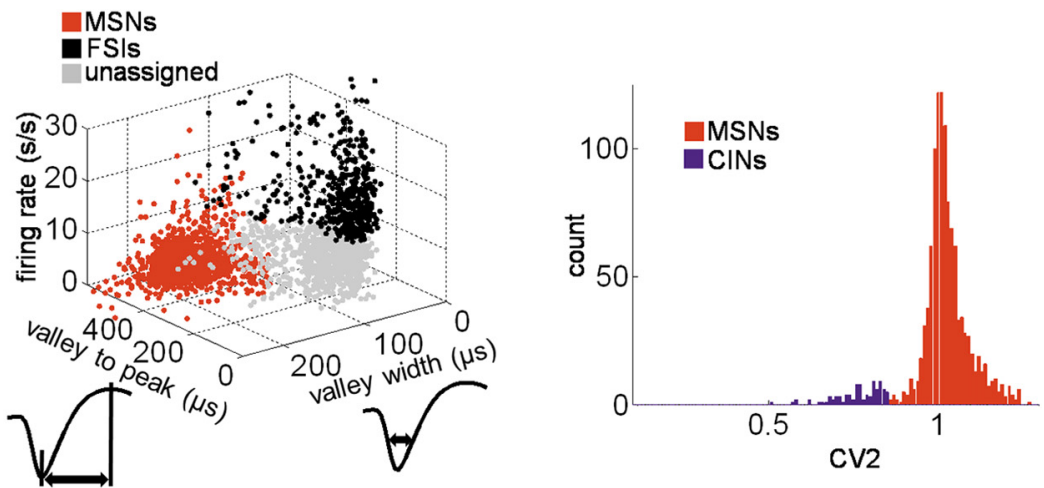

\section{b Average Waveforms}

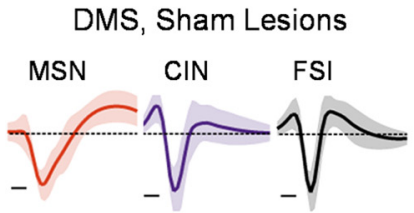

DLS, Sham Lesions

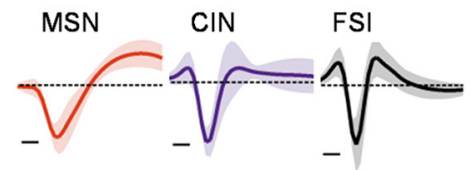

DMS, Unilateral OFC Lesions

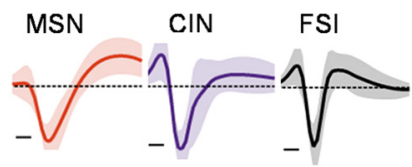

DLS, Unilateral OFC Lesions

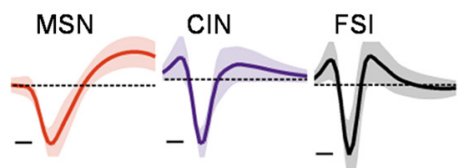

Figure 2. Cell-type separation. $\boldsymbol{a}, \mathrm{MSN}$ s were first separated from other neurons [including fast-spiking interneurons (FSIs)] using a three-dimensional cluster analysis on all recorded units (left). Subsequently, putative CINs were selected by taking the average spike-to-spike CV2 of every unit's entire spike-train. The criteria for CINs was CV2 $<0.85$ and average firing rate $<8$ spikes/s, which were based on measurements from histochemically confirmed CINs reported in published juxtacellular recordings. These criteria effectively discriminated CINs from separately defined MSNs (right; CV2 distribution, low-firing rate MSNs versus putative (INs). The upper limit of the CIN criterion is outside of the $1-\alpha$ confidence interval for low-firing rate MSNs with $\alpha=0.028$. $\boldsymbol{b}$, Resulting average waveforms for each cell type in each recording group (the dotted line represents the 0 voltage level. Scale bar, $100 \mu$ s. Shading represents SE). Note that CINs were not selected based on waveform, whereas MSNs and FSIs were. FSls were not analyzed for this report. 


\section{a DMS CIN Population Block-Selectivity}

preferred direction

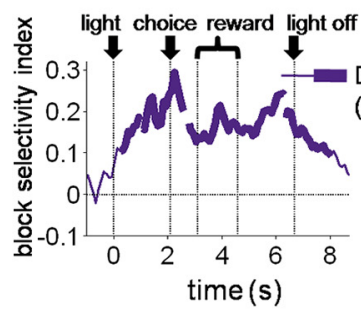

anti-preferred direction

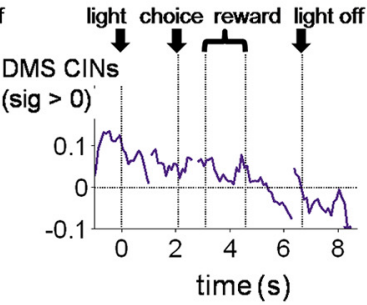

C DMS CIN Unit Block-Selectivity

Pre-odor vs. Post-reward $\quad \mathrm{R}^{2}$ for Other Epochs
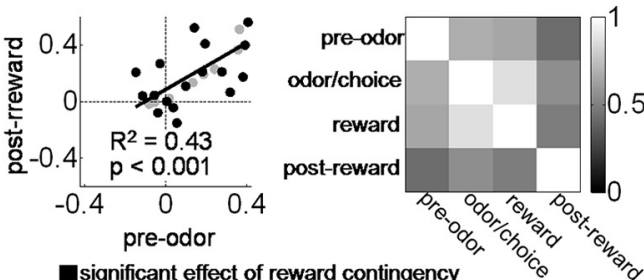

all othe

\section{b DLS CIN Population Block Selectivity}

preferred direction

anti-preferred direction
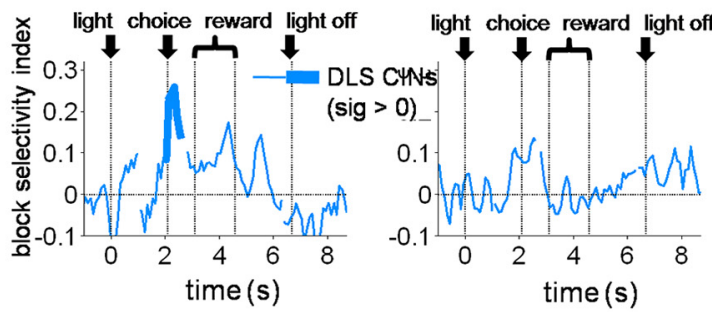

\section{d DLS CIN Unit Block-Selectivity}

\section{Pre-odor vs. Post-reward $\quad \mathrm{R}^{2}$ for Other Epochs}

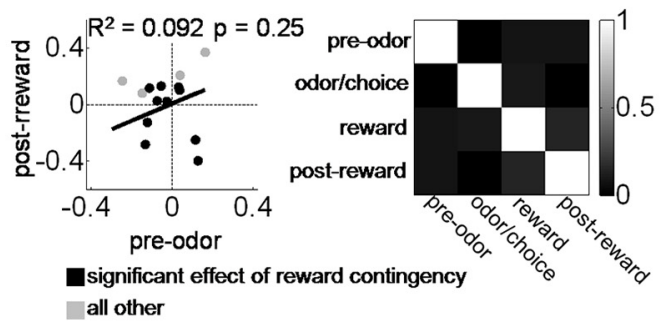

Figure 3. DMS CINs track the current state (i.e., block) across trials. Plots in $\boldsymbol{a}$ and $\boldsymbol{b}$ show a bin-by-bin average index of block-selectivity for the entire DMS ( $n=26)$ and DLS ( $n=16)$ CIN populations, respectively, averaged by each neuron's preferred direction (defined as direction with highest average firing rate during the choice movement; left) or anti-preferred direction (right). Only correct forced-choice trials after the first 20 correct trials in blocks were included in these averages. Bins were aligned to multiple trial events separated by the average time between them. Block-selectivity scores of individual DMS CINs were strongly correlated between different epochs across the trial (c), but this was not true of DLS CINs (d). c, d, Left, Scores for pre-odor epoch versus post-reward epoch; other pairs of epochs are summarized on the right. All $R^{2}$ values for DMS CINs were significant at $p<0.001$, average $R^{2}=0.61$. For DLS CINs, none of thx $R^{2}$ values were significant at $p<0.05$ ( $p$ values $>0.13$ ); average $R^{2}=0.07$

\section{Decoding of Block across Trial CINs vs. MSNs}
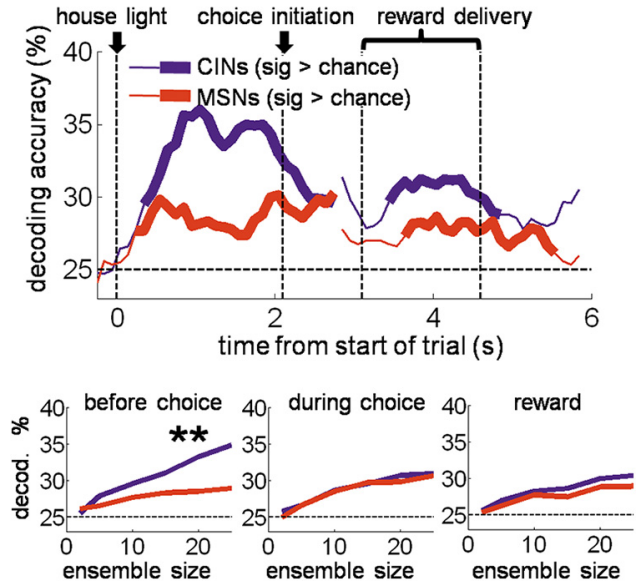

Figure 4. DMS CIN pseudo-ensembles decode the current state (i.e., block) across the trials. The top plot shows block decoding accuracy of a pseudo-ensemble of 25 DMS CINs versus MSNs across the trial. All rewarded trials after the first 20 correct trials in blocks were used in the decoder, regardless of direction. Bottom plots show decoding accuracy as a function of ensemble size for three 1 s epochs across the trial. ${ }^{* *} p<0.01$. 
a

Correct Decoding of Block on Suboptimal Choices: CINs vs. MSNs

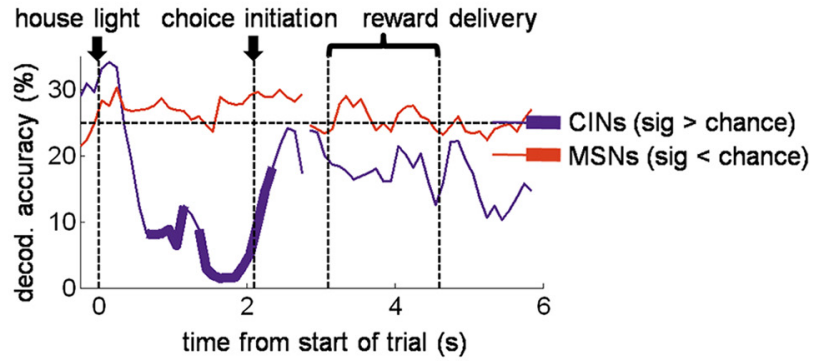

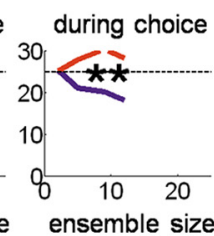

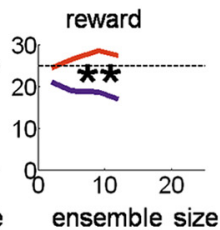

\section{b Miscoding of Block as Opposite Number Block on} Suboptimal Choices: CINs vs. MSNs

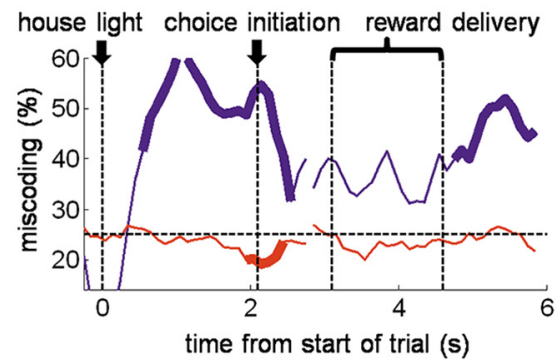

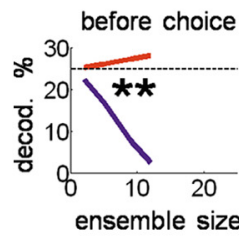

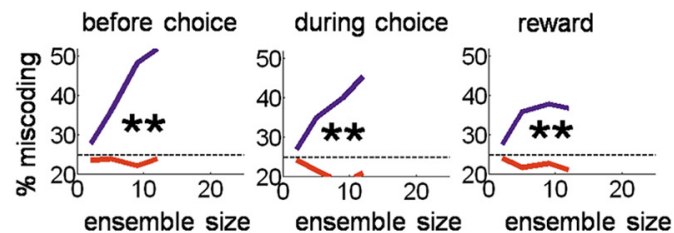

\section{Reaction Time on Suboptimal Choices was Abnormally Fast}

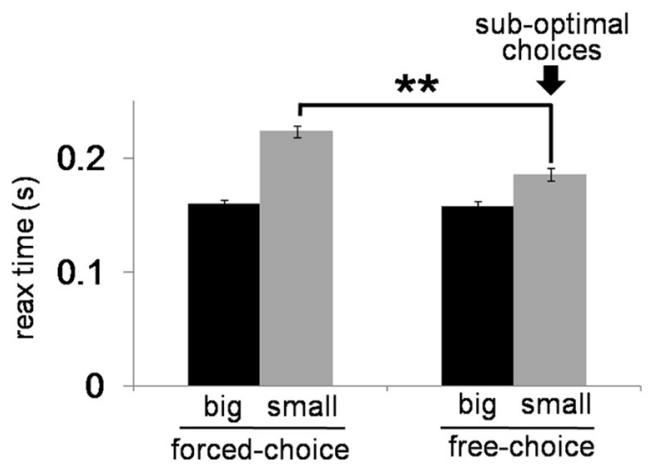

Figure 5. On suboptimal (i.e., inappropriate) choices, DMS CINs miscoded the block as the one that would have been appropriate for that choice. Top plots show percentage accurate block decoding $(\boldsymbol{a})$ and percentage miscoding as the opposite drop-number block $(\boldsymbol{b})$, by a pseudo-ensemble of 12 CINs or MSNs on suboptimal choices. Twelve CINs were recorded in sessions with enough such free-choices. Bottom plots show accurate decoding and miscoding as a function of ensemble size for three $1 \mathrm{~s}$ epochs across the trial. c, Bars show average ( $\pm S E$ ) reaction time by trial type. Reaction times were faster for big reward than small reward, showing that faster reaction times occurred when more valuable rewards were predicted. However, free-choices of the small reward, suboptimal choices, were still significantly faster than forced-choices of the small reward, suggesting an inaccurate (and perhaps optimistic) prediction as to the number of drops to be received on those trials. ${ }^{* *} p<0.01$. 


\section{a Correct Decoding of Block by CINs Optimal vs. Suboptimal Choices}

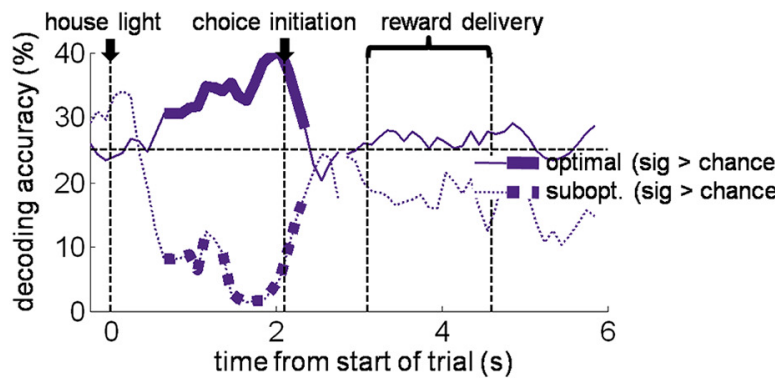

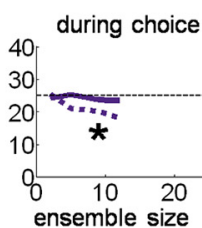

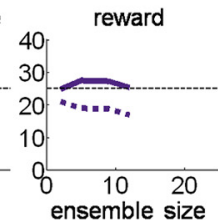

\section{Correct Decoding of Block by MSNs Optimal vs. Suboptimal Choices}

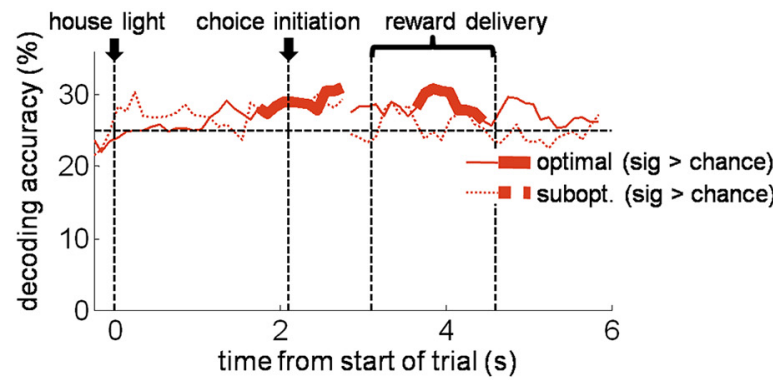

b Miscoding of Block as Opposite Number Block on Optimal vs. Suboptimal Choices

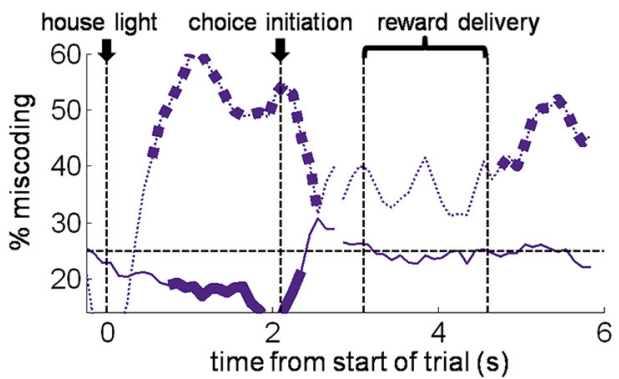

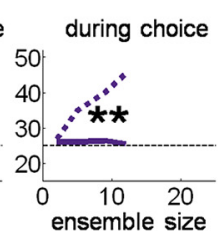

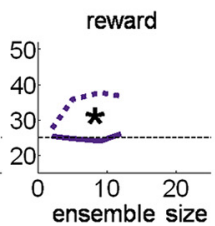

\section{d Miscoding of Block as Opposite Number Block on Optimal vs. Suboptimal Choices}
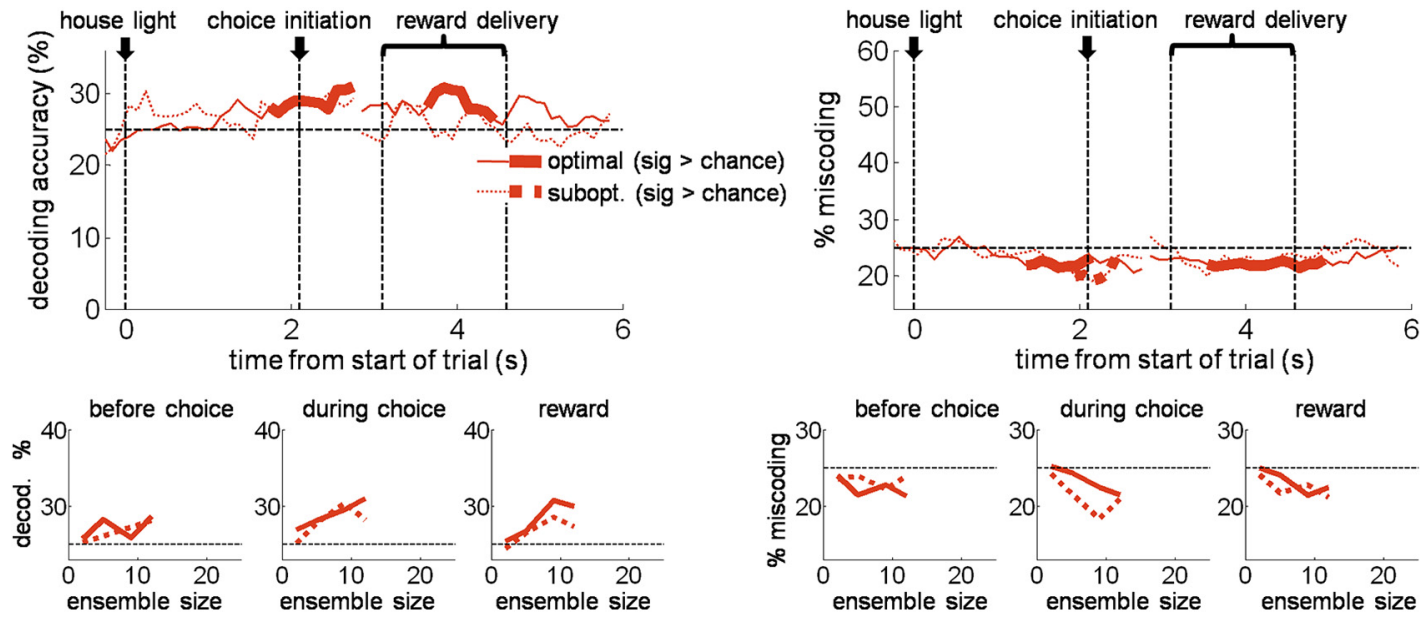

Figure 6. Decoder performance on free-choices of the big reward (i.e., optimal choices) compared with that on free-choices of the small reward (i.e., suboptimal choices). $\boldsymbol{a}, \boldsymbol{c}$, Top plots show block decoding accuracy of a pseudo-ensemble of $12 \mathrm{DMSCINs}$ (a) or $12 \mathrm{DMSMSNs}(\boldsymbol{c})$, during sliding $500 \mathrm{~ms}$ epochs across the trial. The same procedures were used as for the analysis shown in Figure 5, except that only free-choices of the big reward were tested. For comparison, data from small free-choices (suboptimal choices), shown in Figure 5 , is also plotted here in dotted lines. Bottom plots show decoding accuracy as a function of ensemble size for the same three epochs shown in Figure $4 . \boldsymbol{b}, \boldsymbol{d}$, Top plots show percentage of test trials in which the block decoder from the corresponding plot (in $\boldsymbol{a}$ and $\boldsymbol{c}$, respectively) misclassified the block as the one with the opposite drop-number rewards but the same flavor. Bottom plots show the misclassification percentage as a function of ensemble size. ${ }^{*} p<0.05^{* *} p<0.01$. 
a

Decoding of Block by MSNs

On Suboptimal Choices
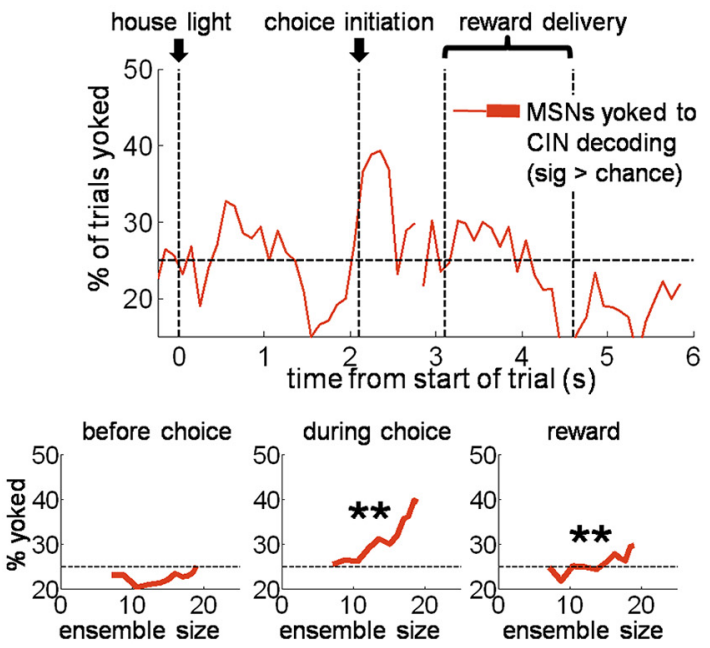

C

Decoding of Block by MSNs

Yoked to CIN Block Decoding

On Optimal Choices
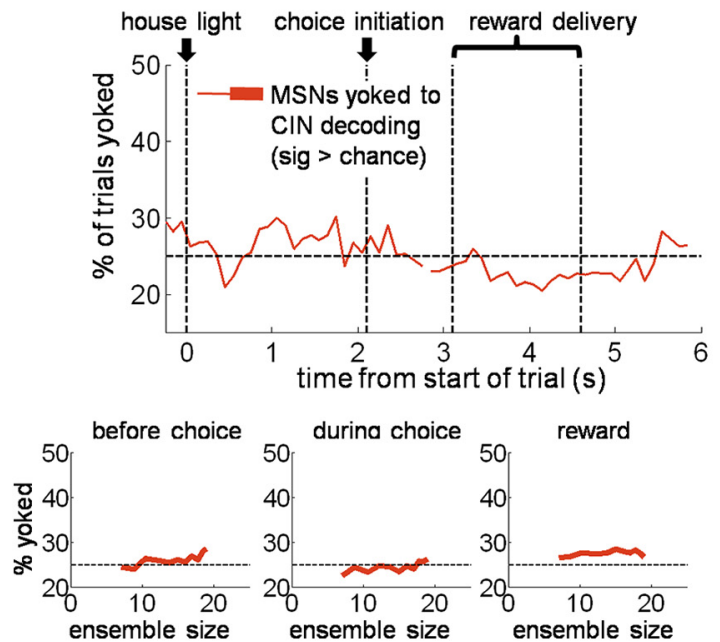

b

\section{Decoding of Block by MSNs \\ On Suboptimal Choices}
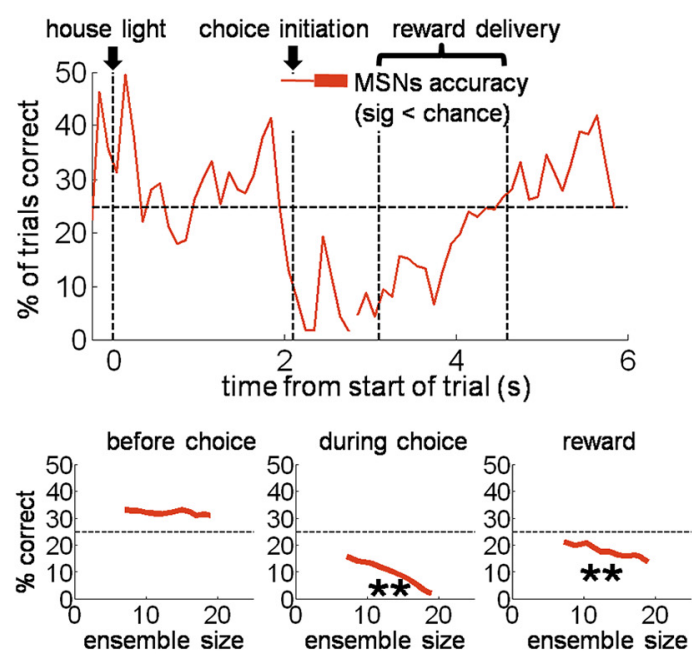

d

\section{Decoding of Block by MSNs \\ On Optimal Choices}
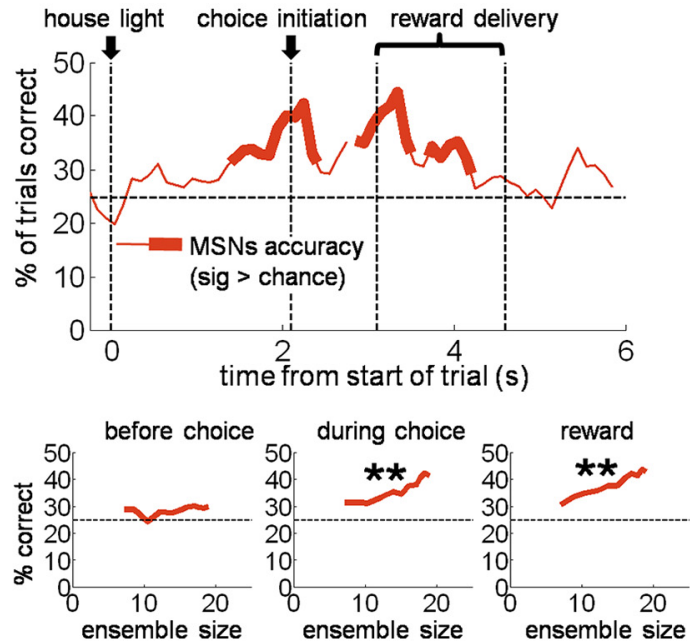

Figure 7. MSNs block decoding is "yoked" to CIN decoding during execution of suboptimal choices. $\boldsymbol{a}$ - $\boldsymbol{d}$, Top plots show block decoding accuracy of pseudo-ensembles of MSNs recorded in the same sessions as CINs. a, c, MSN decoding yoked to CIN decoding on suboptimal choices, meaning the percentage of trials on which MSN ensembles identified the same block as the CIN ensembles. $\boldsymbol{b}, \boldsymbol{d}$, Block decoding accuracy of the same MSN ensembles as $\boldsymbol{a}$ and $\boldsymbol{c}$ (i.e., not yoked to (INs). Bottom plots show decoding accuracy as a function of ensemble size for three $500 \mathrm{~ms}$ epochs across the trial. (starting $1000 \mathrm{~ms}$ before choice initiation, $100 \mathrm{~ms}$ before choice initiation, and at first reward delivery, respectively). ${ }^{* *} p<0.01$ compared with 0 . 


\section{Decoding of Choice Direction on Free-choice Trials}
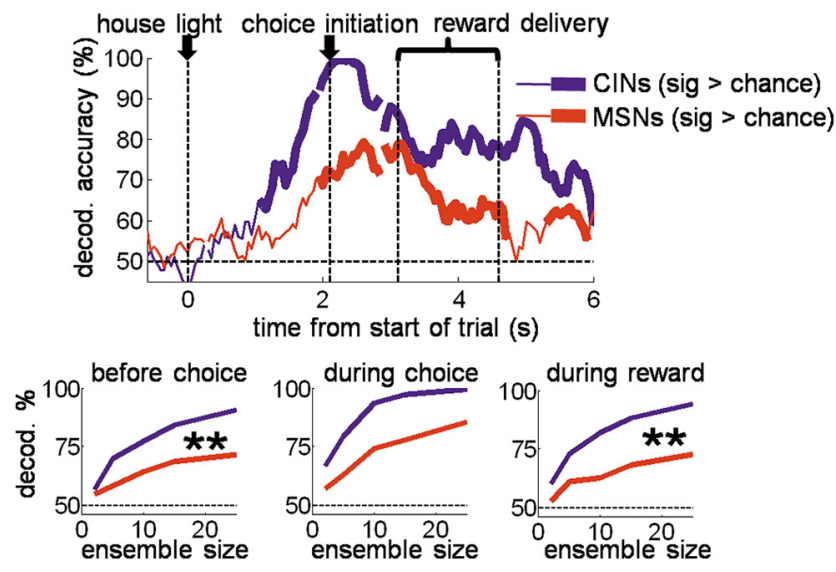

Figure 8. DMS CINs also coded the direction of free-choice before and after it occurred better than MSNs. Top plot shows choice-direction decoding accuracy of a pseudo-ensemble of 25 CINs or MSNs. Only free-choice trials across entire sessions were used; therefore significant decoding of direction before the choice could only reflect an internal intention or state. Bottom plots show decoding accuracy as a function of ensemble size for three 1 s epochs across the trial. ${ }^{* *} p<0.001$.

\section{a \\ DMS CIN Population Block-Selectivity CINs/sham lesion vs. CINs/OFC lesion}

preferred direction

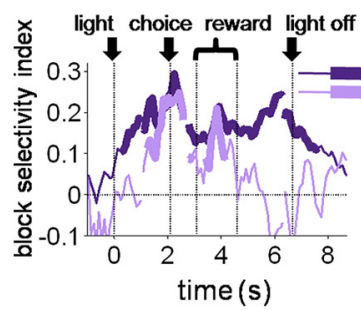

C

DMS CIN Unit Block-Selectivity: Ipsilateral OFC Lesions

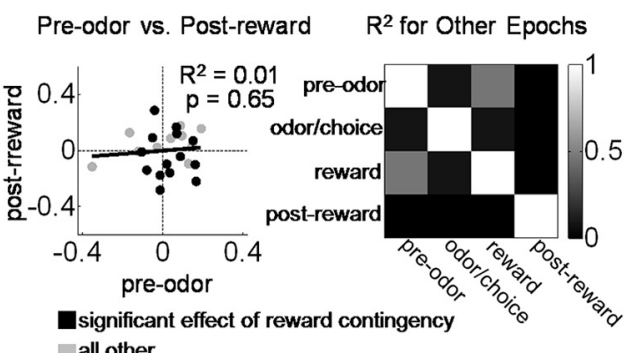

b DLS CIN Population Block Selectivity CINs/sham lesion vs. CINs/OFC lesion

preferred direction

anti-preferred direction
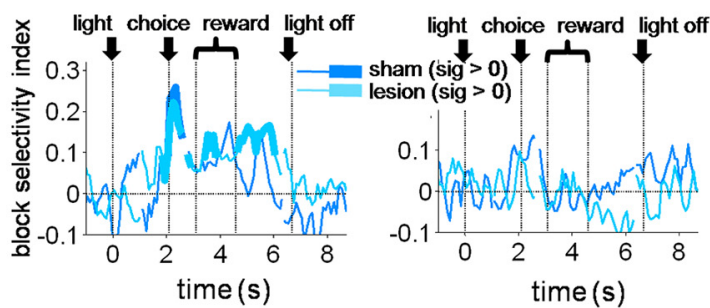

d

DLS CIN Unit Block-Selectivity: Ipsilateral OFC Lesions

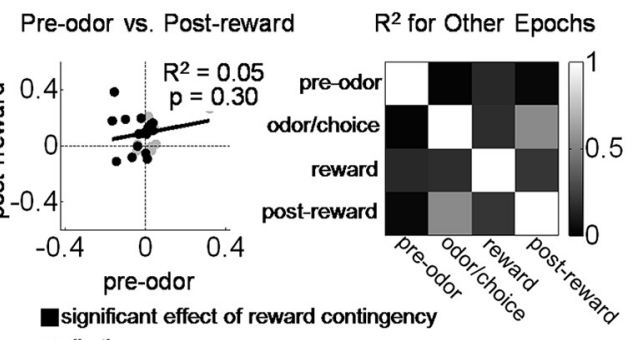

all other

Figure 9. OFC lesions eliminate state encoding in DMS CINs. As in Figure 3, plots in $\boldsymbol{a}$ and $\boldsymbol{b}$ show a bin-by-bin average index of block-selectivity for the entire DMS ( $\boldsymbol{a}$ ) and DLS (b) CIN population in OFC-lesioned rats compared with those in sham rats. Block-selectivity scores of individual CINs in cand $\boldsymbol{d}$ show that OFC lesions eliminated the consistency of block-selectivity across the trial in DMS CINs (c) without affecting that in DLS CINs (d). Left, Scores in a pre-odor epoch versus post-reward epoch; other pairs of epochs are summarized on the right. 

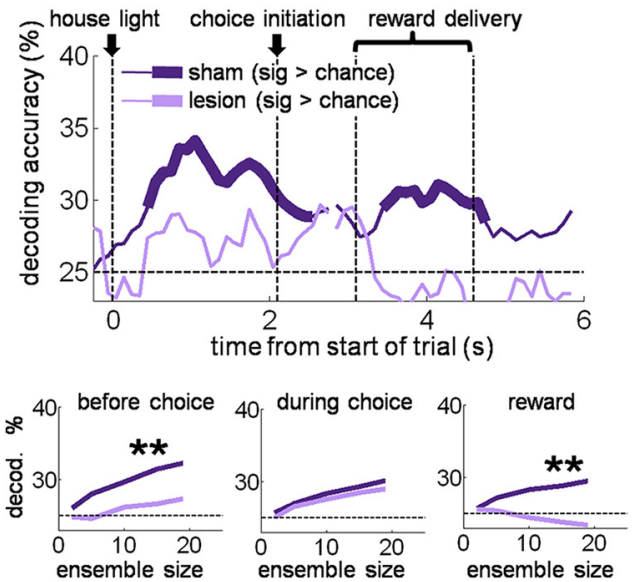

Figure 10. OFC lesions eliminate state encoding in DMS CINs. As in Figure 4, top plot shows block decoding accuracy of a pseudo-ensemble of 19 DMS CINs in OFC-lesioned rats (only 19 neurons were recorded in blocks with a sufficient number of trials) versus those in sham rats, also with a pseudo-ensemble of 19 neurons for comparison. Bottom plots show decoding accuracy as a function of ensemble size for three $1 \mathrm{~s}$ epochs across the trial. ${ }^{* *} p<0.01$.

a
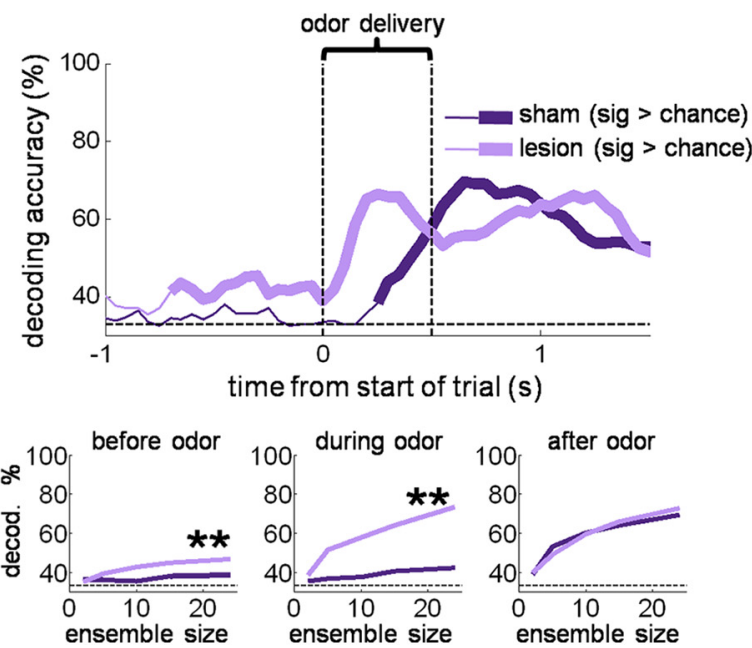

Decoding of Odor Identity CINs/sham vs. CINs/OFC lesion

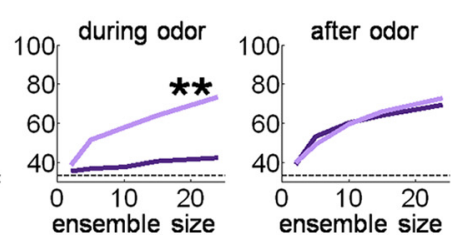

b Decoding of Number of Drops
CINs/sham vs. CINs/OFC lesion
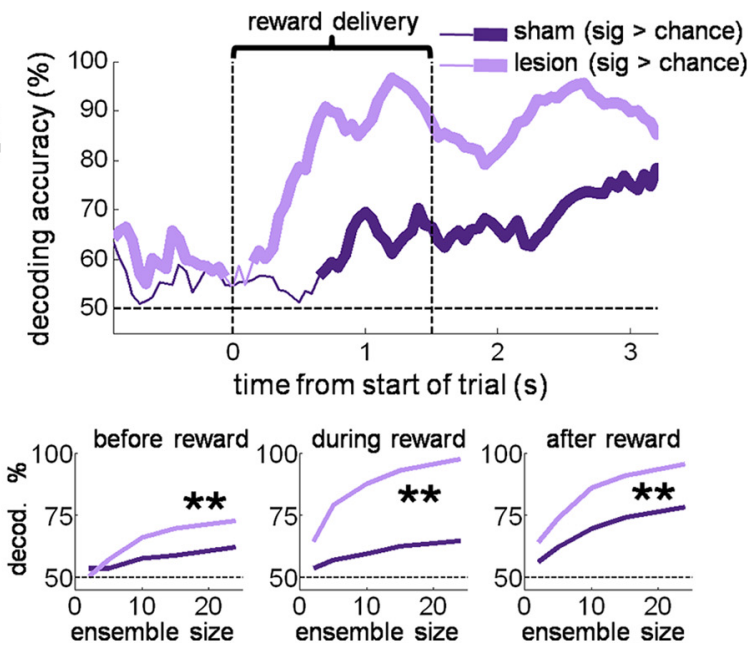
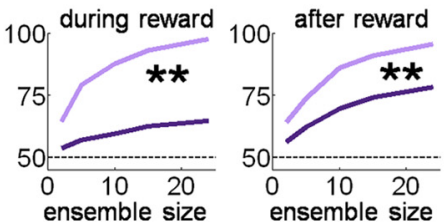

Figure 11. DMS CINs recorded in rats with OFC lesions decode trial stimuli better than those in sham rats. Top plots show odor identity $(\boldsymbol{a})$ and reward number (b) decoding accuracy of a pseudo-ensemble of 24 DMS CINs in shams versus OFC lesions, during sliding $200 \mathrm{~ms}$ epochs across the trial. All correct trials were included and therefore for odor identity, three odors had to be decoded (the forced-choice left, forced-choice right, and free-choice odors). Note that because of correction trials and the pseudorandom sequence of trials, some limited information about the upcoming trial-type could be derived before odor delivery began. Bottom plots show decoding accuracy as a function of ensemble size for three epochs each: for odor decoding, $1 \mathrm{~s}$ ending with the beginning of odor delivery, the $500 \mathrm{~ms}$ of odor delivery, and the $1 \mathrm{~s}$ immediately following odor delivery; for reward number decoding, $1 \mathrm{~s}$ ending with the beginning of reward delivery, the period of reward delivery and consumption ( $1500 \mathrm{~ms}$ beginning with delivery of the first drop of reward), and $1 \mathrm{~s}$ immediately after the end of the reward delivery epoch. ${ }^{* *} p<0.01$. 


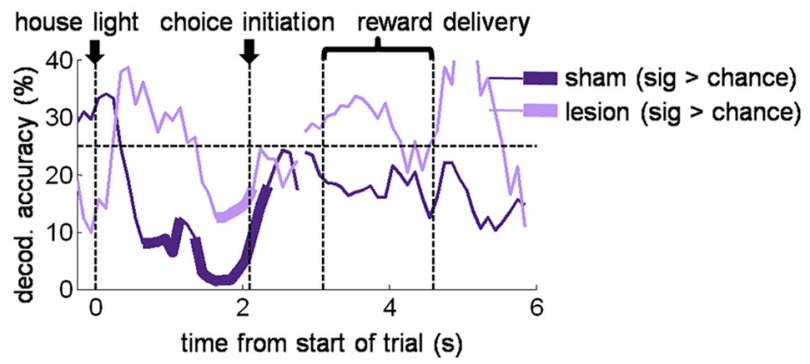

\section{a Correct Decoding of Block on Suboptimal Choices: CINs/sham vs. CINs/OFC lesion}

\section{b Miscoding of Block as Opposite Number Block on Suboptimal Choices:}
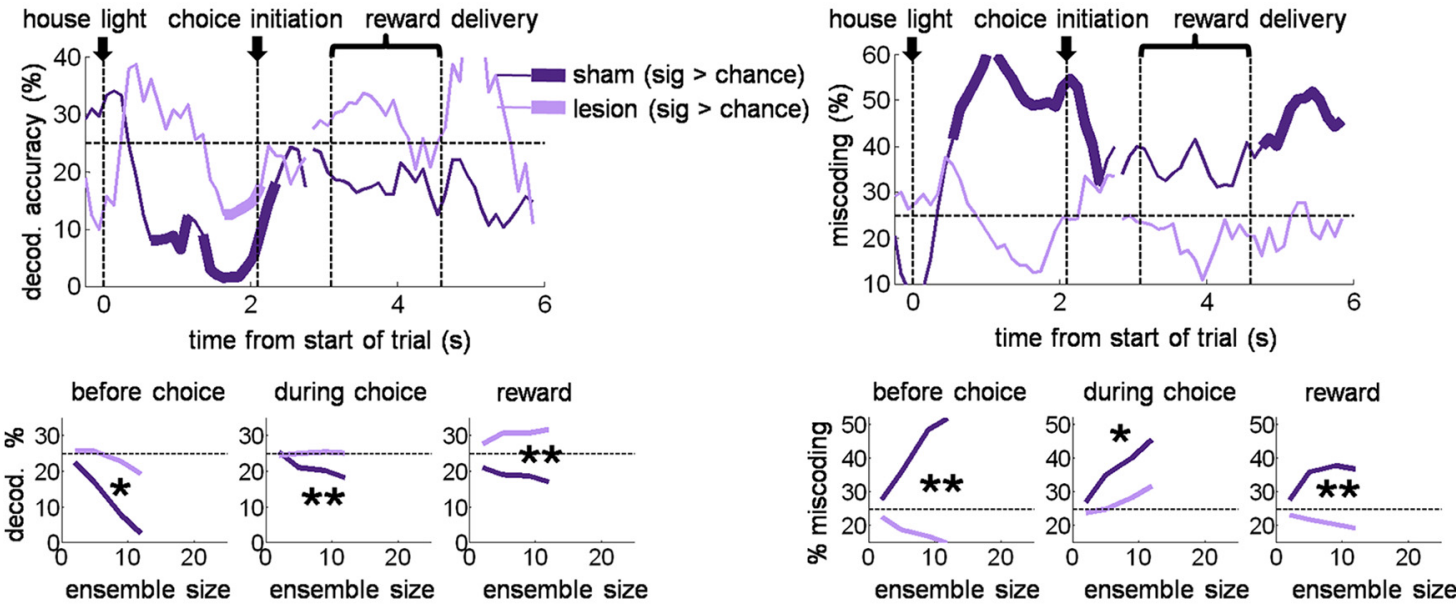

C

Reaction Time on Suboptimal Choices In Lesioned Rats

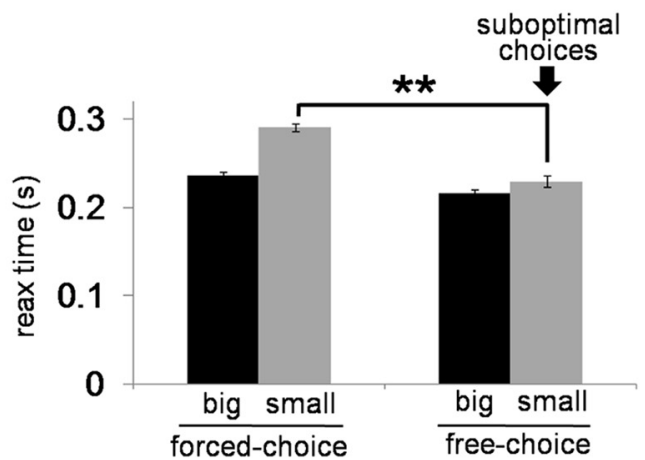

\section{d Decoding of Choice Direction on Free-choice Trials}
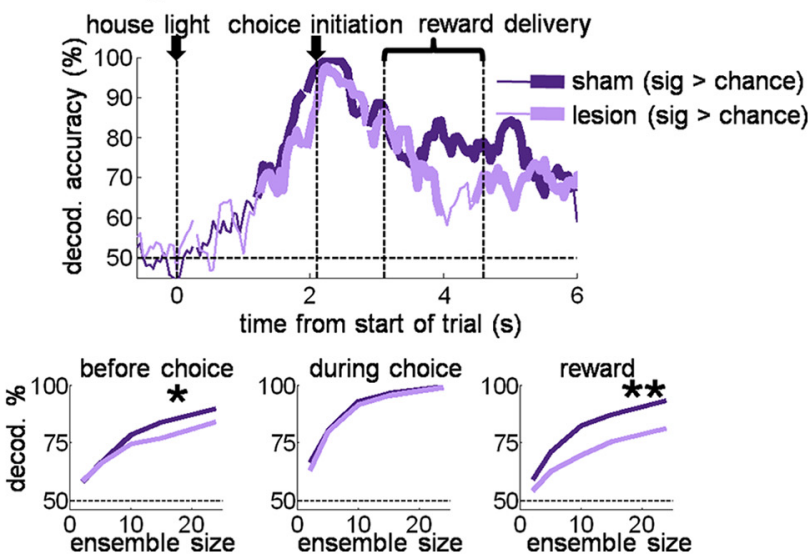

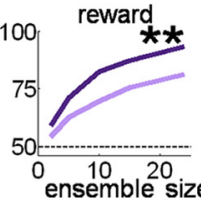

Figure 12. Ipsilateral OFC lesions eliminate the relationship between free-choices of the small reward and miscoding of the block in DMS CINs. Top plots show percentage accurate block decoding (a) and percentage miscoding as the opposite drop-number block $(\boldsymbol{b})$, by a pseudo-ensemble of $12 \mathrm{CINs}$ in sham rats or ipsilateral OFC-lesioned rats on suboptimal choices. Bottom plots show accurate decoding and miscoding as a function of ensemble size for three $1 \mathrm{~s}$ epochs across the trial. $c$, Bars show average ( \pm SE) reaction time by trial type in lesioned rats. The pattern of reaction times was the same as in sham rats (Fig. 5); that is, they were faster for big reward than small reward, but free-choices of the small reward, suboptimal choices, were similarly fast to those when a big reward was expected, suggesting an inaccurate prediction as to the number of drops to be received on those trials. $\boldsymbol{d}$, Top plot shows choice-direction decoding accuracy of a pseudo-ensemble of 24 CINs in sham rats or ipsilateral OFC-lesioned rats. Only free-choice trials were used; therefore significant decoding of direction before the choice could only reflect an internal intention or state. Bottom plots show decoding accuracy as a function of ensemble size for three 1 s epochs across the trial. ${ }^{* *} p<0.01$. 